\title{
Research Article \\ Delay-Dependent Exponential Stability for Discrete-Time BAM Neural Networks with Time-Varying Delays
}

\author{
Yonggang Chen, ${ }^{1}$ Weiping $\mathrm{Bi}^{,}{ }^{2}$ and Yuanyuan $\mathrm{Wu}^{3}$ \\ ${ }^{1}$ Department of Mathematics, Henan Institute of Science and Technology, Xinxiang 453003, China \\ ${ }^{2}$ College of Mathematics and Information Science, Henan Normal University, Xinxiang 453007, China \\ ${ }^{3}$ Key Laboratory of Measurement and Control of Complex Systems of Engineering, Ministry of Education, \\ School of Automation, Southeast University, Nanjing 210096, China
}

Correspondence should be addressed to Yonggang Chen, happycygzmd@tom.com

Received 18 May 2008; Revised 5 August 2008; Accepted 10 September 2008

Recommended by Yong Zhou

This paper considers the delay-dependent exponential stability for discrete-time BAM neural networks with time-varying delays. By constructing the new Lyapunov functional, the improved delay-dependent exponential stability criterion is derived in terms of linear matrix inequality (LMI). Moreover, in order to reduce the conservativeness, some slack matrices are introduced in this paper. Two numerical examples are presented to show the effectiveness and less conservativeness of the proposed method.

Copyright (C) 2008 Yonggang Chen et al. This is an open access article distributed under the Creative Commons Attribution License, which permits unrestricted use, distribution, and reproduction in any medium, provided the original work is properly cited.

\section{Introduction}

Bidirectional associative memory (BAM) neural networks were first introduced by Kosko [1, 2]. It generalizes the single-layer autoassociative Hebbian correlator to a two-layer patternmatched heteroassociative circuits. This class of neural networks has been successfully applied to pattern recognition and artificial intelligence. In those applications, it is very important to guarantee the designed neural networks to be stable. On the other hand, time delays are unavoidably encountered in the implementation of neural networks, and its existence will lead to instability, oscillation, and poor performances of neural networks. Therefore, the the asymptotic or exponential stability analysis for BAM neural networks with time delays has received great attention during the past years, see, for example, [3-15]. The obtained results are classified into two categories: delay-independent results $[3-5,7,10]$ and delay-dependent results $[6,9,11,12,14-16]$. Generally speaking, the delay-dependent results are less conservative than the delay-independent ones, especially when the size of time delay is small. 
It should be pointed out that all of the above-mentioned references are concerned with continuous-time BAM neural networks. However, when implementing the continuous-time neural networks for computer simulation, for experimental or computational purposes, it is essential to formulate a discrete-time system that is an analogue of the continuous-time recurrent neural networks [17]. Generally speaking, the stability analysis of continuous-time neural networks is not applicable to the discrete version. Therefore, the stability analysis for various discrete-time neural networks with time delays is widely studied in recent years [17-28]. Using M-matrix method, Liang and Cao [25] studied the exponential stability of continuous-time BAM neural network with constant delays and its discrete analogue. Liang et al. [26] also studied the dynamics of discrete-time BAM neural networks with variable time delays based on unreasonably severe constraints on the delay functions. By using the Lyapunov functional method and linear matrix inequality technique, the exponential stability and robust exponential stability for discrete-time BAM neural networks with variable delays were considered in $[27,28]$, respectively. However, the results presented in $[27,28]$ are still conservative, since the the Lyapunov functionals constructed in $[27,28]$ are so simple. Therefore, there is much room to improve the results in $[27,28]$.

In this paper, we present a new exponential stability criterion for discrete-time BAM neural networks with time-varying delays. Compared with the existing methods, the main contributions of this paper are as follows. Firstly, more general Lyapunov functional is employed to obtain the improved exponential stability criterion; secondly, in order to reduce the conservativeness, some slack matrices, which bring much flexibility in solving LMI, are introduced in this paper. The proposed exponential stability criterion is expressed in LMI which can be efficiently solved by LMI toolbox in Matlab. Finally, two numerical examples are presented to show that our result is less conservative than some existing ones $[27,28]$.

The organization of this paper is as follows. Section 2 presents problem formulation of discrete-time BAM neural networks with time delay-varying. In Section 3, our main result of this paper is established and some remarks are given. In Section 4, numerical examples are given to demonstrate the proposed method. Finally, conclusion is drawn in Section 5.

\section{Notation}

Throughout this paper, the superscript " $T$ " stands for the transpose of a matrix. $R^{n}$ and $R^{n \times n}$ denote the $n$-dimensional Euclidean space and set of all $n \times n$ real matrices, respectively. A real symmetric matrix $P>0(\geq 0)$ denotes $P$ being a positive definite (positive semidefinite) matrix. $I$ is used to denote an identity matrix with proper dimension. For integers $a, b$, and $a<b, N[a, b]$ denote the discrete interval $N[a, b]=\{a, a+1, \ldots, b-1, b\} . \lambda_{M}(X)$ and $\lambda_{m}(X)$ stand for the maximum and minimum eigenvalues of the symmetric matrix $X$, respectively. Matrices, if not explicitly stated, are assumed to have compatible dimensions. The symmetric terms in a symmetric matrix are denoted by $*$.

\section{Problem formulation}

Consider the following discrete-time BAM neural network with time-varying delays:

$$
\begin{aligned}
& u_{i}(k+1)=a_{i} u_{i}(k)+\sum_{j=1}^{m} w_{i j} \tilde{f}_{j}\left(v_{j}(k-\tau(k))\right)+I_{i}, \quad i=1,2, \ldots, n, \\
& v_{j}(k+1)=b_{j} v_{j}(k)+\sum_{i=1}^{n} v_{j i} \tilde{g}_{i}\left(u_{i}(k-h(k))\right)+J_{j}, \quad j=1,2, \ldots, m,
\end{aligned}
$$


where $u_{i}(k)$ and $v_{j}(k)$ are the states of the $i$ th neuron from the neural field $F_{X}$ and the $j$ th neuron from the neural field $F_{Y}$ at time $k$, respectively; $a_{i}, b_{j} \in(0,1)$ denote the stability of internal neuron process on the $X$-layer and the $Y$-layer, respectively; $w_{i j}, v_{j i}$ are real constants, and denote the synaptic connection weights; $\tilde{f}_{j}(\cdot) \tilde{g}_{i}(\cdot)$ denote the activation functions of the $j$ th neuron from the neural field $F_{Y}$ and the $j$ th neuron from the neural field $F_{X}$, respectively; $I_{i}$ and $J_{j}$ denote the external constant inputs from outside the network acting on the $i$ th neuron from the neural field $F_{X}$ and the $j$ th neuron from the neural field $F_{Y}$, respectively. $\tau(k)$ and $h(k)$ represent time-varying interval delays satisfying

$$
\tau_{m} \leq \tau(k) \leq \tau_{M}, \quad h_{m} \leq h(k) \leq h_{M},
$$

where $\tau_{m}, \tau_{M}, h_{m}$, and $h_{M}$ are positive integers.

Throughout this paper, we make the following assumption on the activation functions $\tilde{f}_{j}(\cdot), \tilde{g}_{i}(\cdot)$.

(A1) The activation functions $\tilde{f}_{j}(\cdot), \tilde{g}_{i}(\cdot)(i=1,2, \ldots, n, j=1,2, \ldots, m)$ are bounded on $R$.

(A2) For any $\zeta_{1}, \zeta_{2} \in R$, there exist positive scalars $l_{1 j}, l_{2 i}$ such that

$$
\begin{array}{ll}
0 \leq\left|\tilde{f}_{j}\left(\zeta_{1}\right)-\tilde{f}_{j}\left(\zeta_{2}\right)\right| \leq l_{1 j}\left|\zeta_{1}-\zeta_{2}\right|, & j=1,2, \ldots, m, \\
0 \leq\left|\tilde{g}_{j}\left(\zeta_{1}\right)-\tilde{g}_{j}\left(\zeta_{2}\right)\right| \leq l_{2 i}\left|\zeta_{1}-\zeta_{2}\right|, & i=1,2, \ldots, n .
\end{array}
$$

It is clear that under Assumption (A1) and (A2), system (2.1) has at least one equilibrium. In order to simplify our proof, we shift the equilibrium point $u^{*}=\left[u_{1}^{*}, u_{2}^{*}\right.$, $\left.\ldots, u_{n}^{*}\right]^{T}, v^{*}=\left[v_{1}^{*}, v_{2}^{*}, \ldots, v_{m}^{*}\right]^{T}$ of system (2.1) to the origin. Let $x_{i}(k)=u_{i}(k)-u_{i}^{*}, y_{j}(k)=$ $v_{j}(k)-v_{j}^{*}, f_{j}\left(y_{j}(k)\right)=\tilde{f}_{j}\left(v_{j}(k)\right)-\tilde{f}_{j}\left(v_{j}^{*}\right), g_{i}\left(x_{i}(k)\right)=\tilde{g}_{i}\left(u_{i}(k)\right)-\tilde{g}_{i}\left(u_{i}^{*}\right)$, then system $(2.1)$ can be transformed to

$$
\begin{array}{ll}
x_{i}(k+1)=a_{i} x_{i}(k)+\sum_{j=1}^{m} w_{i j} f_{j}\left(y_{j}(t-\tau(k))\right), & i=1,2, \ldots, n \\
y_{j}(k+1)=b_{j} y_{j}(k)+\sum_{i=1}^{n} v_{j i} g_{i}\left(x_{i}(t-h(k))\right), & j=1,2, \ldots, m .
\end{array}
$$

Obviously, the the activation functions $f_{j}(\cdot), g_{i}(\cdot)$ satisfy the following conditions.

(A3) For any $\zeta \in R$, there exist positive scalars $l_{1 j}, l_{2 i}$ such that

$$
0 \leq\left|f_{j}(\zeta)\right| \leq l_{1 j}|\zeta|, \quad j=1,2, \ldots, m, \quad 0 \leq\left|g_{i}(\zeta)\right| \leq l_{2 i}|\zeta|, \quad i=1,2, \ldots, n .
$$

Also we assume that system (2.4) with initial value

$$
x_{i}(s)=\phi_{i}(s), \quad y_{j}(s)=\psi_{j}(s), \quad \forall s \in N\left[-\tau^{*}, 0\right], i=1,2, \ldots, n, j=1,2, \ldots, m,
$$

where $\tau^{*}=\max \left\{\tau_{M}, h_{M}\right\}$. 
For convenience, we rewrite system (2.4) in the form

$$
\begin{gathered}
x(k+1)=A x(k)+W f(y(k-\tau(k))), \\
y(k+1)=B y(k)+V g(x(k-h(k))), \\
x(s)=\phi(s), \quad y(s)=\psi(s), \quad \forall s \in N\left[-\tau^{*}, 0\right],
\end{gathered}
$$

where $x(k)=\left[x_{1}(k), x_{2}(k), \ldots, x_{n}(k)\right]^{T}, y(k)=\left[y_{1}(k), y_{2}(k), \ldots, y_{m}(k)\right]^{T}, f(y(k))=$ $\left[f_{1}\left(y_{1}(k)\right), f_{2}\left(y_{2}(k)\right), \ldots, f_{m}\left(y_{m}(k)\right)\right]^{T}, g(x(k))=\left[g_{1}\left(x_{1}(k)\right), g_{2}\left(x_{2}(k)\right), \ldots, g_{n}\left(x_{n}(k)\right)\right]^{T}, A=$ $\operatorname{diag}\left\{a_{1}, a_{2}, \ldots, a_{n}\right\}, B=\operatorname{diag}\left\{b_{1}, b_{2}, \ldots, b_{m}\right\}, W=\left(w_{i j}\right)_{m \times n}, V=\left(v_{i j}\right)_{n \times m}, \phi(s)=\left[\phi_{1}(s)\right.$, $\left.\phi_{2}(s), \ldots, \phi_{n}(s)\right]^{T}, \psi(s)=\left[\psi_{1}(s), \psi_{2}(s), \ldots, \psi_{m}(s)\right]^{T}$.

From above analysis, we can see that the exponential stability problem of system (2.1) on equilibrium $x^{*}$ is changed into the zero stability problem of system (2.7). Therefore, in the following part, we will devote into the exponential stability analysis problem of system (2.7). lemmas.

Before giving the main result, we will firstly introduce the following definition and

Definition 2.1. The trivial solution of BAM neural network (2.7) is said to be globally exponentially stable, if there exist scalars $r>1$ and $M>1$ such that

$$
\|x(k)\|^{2}+\|y(k)\|^{2} \leq M\left(\sup _{s \in N\left[-h_{M}, 0\right]}\|\phi(s)\|^{2}+\sup _{s \in N\left[-\tau_{M}, 0\right]}\|\psi(s)\|^{2}\right) r^{-k}
$$

Lemma 2.2 (see [29]). For any real vectors $a, b$, and any matrix $Q>0$ with appropriate dimensions, it follows that

$$
2 a^{T} b \leq a^{T} Q a+b^{T} Q^{-1} b
$$

\section{Main result}

In this section, we are in a position to present the global exponential stability criterion of system (2.7).

Theorem 3.1. For given diagonal matrices $L_{1}=\operatorname{diag}\left\{l_{11}, l_{12}, \ldots, l_{1 m}\right\}$ and $L_{2}=\operatorname{diag}\left\{l_{21}, l_{22}\right.$, $\left.\ldots, l_{2 n}\right\}$. Under Assumptions (A1) and (A2), the system (2.7) is globally exponentially stable, if there exist matrices $P_{i}>0, Q_{i}>0, R_{i}>0, S_{i}>0, Z_{i}>0,(i=1,2)$, $L_{j}, M_{j}, N_{j}, T_{j},(j=1,2, \ldots, 8)$, and diagonal matrices $D_{1}=\operatorname{diag}\left\{d_{11}, d_{12}, \ldots, d_{1 m}\right\}>0, D_{2}=$ $\operatorname{diag}\left\{d_{21}, d_{22}, \ldots, d_{2 n}\right\}>0$, such that the following LMI holds:

$$
\left[\begin{array}{ccccc}
\Omega & \sqrt{h_{M}} L & \sqrt{h_{M}-h_{m}} M & \sqrt{\tau_{M}} N & \sqrt{\tau_{M}-\tau_{m}} T \\
* & -Z_{1} & 0 & 0 & 0 \\
* & * & -Z_{1} & 0 & 0 \\
* & * & * & -Z_{2} & 0 \\
* & * & * & * & -Z_{2}
\end{array}\right]<0
$$


where

$$
\begin{aligned}
& \omega=\left[\begin{array}{cccccccc}
\omega_{11} & \omega_{12} & \omega_{13} & \omega_{14} & \omega_{15} & \omega_{16} & -m_{1}+l_{7}^{t} & l_{8}^{t} \\
* & \omega_{22} & \omega_{23} & \omega_{24} & \omega_{25} & \omega_{26} & \omega_{27} & t_{8}^{t}-n_{8}^{t} \\
* & * & \omega_{33} & -t_{4}^{t} & \omega_{35} & \omega_{36} & -m_{3}-t_{7}^{t} & -t_{8}^{t} \\
* & * & * & \omega_{44} & n_{4} & m_{4}-l_{4} & -m_{4} & 0 \\
* & * & * & * & \omega_{55} & \omega_{56} & -m_{5}+n_{7}^{t} & \omega_{58} \\
* & * & * & * & * & \omega_{66} & \omega_{67} & -l_{8}^{t}+m_{8}^{t} \\
* & * & * & * & * & * & \omega_{77} & -m_{8}^{t} \\
* & * & * & * & * & * & * & \omega_{88}
\end{array}\right], \\
& l=\left[\begin{array}{llllllll}
l_{1}^{t} & l_{2}^{t} & l_{3}^{t} & l_{4}^{t} & l_{5}^{t} & l_{6}^{t} & l_{7}^{t} & l_{8}^{t}
\end{array}\right]^{t}, \\
& m=\left[\begin{array}{llllllll}
m_{1}^{t} & m_{2}^{t} & m_{3}^{t} & m_{4}^{t} & m_{5}^{t} & m_{6}^{t} & m_{7}^{t} & m_{8}^{t}
\end{array}\right]^{t} \text {, } \\
& n=\left[\begin{array}{llllllll}
n_{1}^{t} & n_{2}^{t} & n_{3}^{t} & n_{4}^{t} & n_{5}^{t} & n_{6}^{t} & n_{7}^{t} & n_{8}^{t}
\end{array}\right]^{t}, \\
& t=\left[\begin{array}{llllllll}
t_{1}^{t} & t_{2}^{t} & t_{3}^{t} & t_{4}^{t} & t_{5}^{t} & t_{6}^{t} & t_{7}^{t} & t_{8}^{t}
\end{array}\right]^{t}, \\
& \omega_{11}=r a^{t} p_{1} a+r^{h_{m}} h_{m}(a-i)^{t} z_{1}(a-i)-p_{1}+\left(h_{m}-h_{m}+1\right) q_{1}+r_{1}+l_{1}+l_{1}^{t} \text {, } \\
& \omega_{12}=l_{2}^{t}+t_{1}-n_{1}, \\
& \omega_{13}=l_{3}^{t}-t_{1}, \\
& \omega_{14}=r a^{t} p_{1} w+r^{h_{m}} h_{m}(a-i)^{t} z_{1} w+l_{4}^{t} \\
& \omega_{15}=l_{5}^{t}+n_{1} \\
& \omega_{16}=-l_{1}+l_{6}^{t}+m_{1} \text {, } \\
& \omega_{22}=-r^{-\tau_{m}} q_{2}+t_{2}+t_{2}^{t}+d_{1}-n_{2}-n_{2}^{t}, \\
& \omega_{23}=-t_{2}+t_{3}^{t}-n_{3}^{t} \\
& \omega_{24}=t_{4}^{t}-n_{4}^{t} \\
& \omega_{25}=t_{5}^{t}+n_{2}-n_{5}^{t}, \\
& \omega_{26}=t_{6}^{t}+m_{2}-l_{2}-n_{6}^{t} \text {, } \\
& \omega_{27}=-m_{2}+t_{7}^{t}-n_{7}^{t} \\
& \omega_{33}=-r^{-\tau_{m}} r_{2}-t_{3}-t_{3}^{t}, \\
& \omega_{35}=n_{3}-t_{5}^{t}
\end{aligned}
$$




$$
\begin{gathered}
\omega_{36}=m_{3}-l_{3}-t_{6}^{t}, \\
\omega_{44}=r w^{t} p_{1} w+r^{h_{m}} h_{m} w^{t} z_{1} w-l_{1}^{-1} d_{1} l_{1}^{-1}, \\
\omega_{55}=r b^{t} p_{2} b+r^{\tau_{m}} \tau_{m}(b-i)^{t} z_{2}(b-i)-p_{2}+\left(\tau_{m}-\tau_{m}+1\right) q_{2}+r_{2}+n_{5}+n_{5}^{t} \\
\omega_{56}=m_{5}-l_{5}+n_{6}^{t}, \\
\omega_{58}=r b^{t} p_{2} v+r^{\tau_{m}} \tau_{m}(b-i)^{t} z_{2} v+n_{8}^{t}, \\
\omega_{66}=-r^{-h_{m}} q_{1}-l_{6}-l_{6}^{t}+d_{2}+m_{6}+m_{6}^{t}, \\
\omega_{67}=-l_{7}^{t}-m_{6}+m_{7}^{t}, \\
\omega_{77}=-r^{-h_{m}} r_{1}-m_{7}-m_{7}^{t}, \\
\omega_{88}=r v^{t} p_{2} v+r^{\tau_{m}} \tau_{m} v^{t} z_{2} v-l_{2}^{-1} d_{2} l_{2}^{-1} .
\end{gathered}
$$

Proof. Choose the following Lyapunov-Krasovskii candidate function of system (2.7) as follows:

$$
V(k)=V_{1}(k)+V_{2}(k)+V_{3}(k)+V_{4}(k)+V_{5}(k),
$$

where

$$
\begin{aligned}
& v_{1}(k)=r^{k} x^{t}(k) p_{1} x(k)+r^{k} y^{t}(k) p_{2} y(k), \\
& v_{2}(k)=\sum_{l=k-h(k)}^{k-1} r^{l} x^{t}(l) q_{1} x(l)+\sum_{l=k-\tau(k)}^{k-1} r^{l} y^{t}(l) q_{2} y(l) \\
& v_{3}(k)=\sum_{\theta=-h_{m}+1}^{-h_{m}} \sum_{l=k+\theta}^{k-1} r^{l} x^{t}(l) q_{1} x(l)+\sum_{\theta=-\tau_{m}+1}^{-\tau_{m}} \sum_{l=k+\theta}^{k-1} r^{l} y^{t}(l) q_{2} y(l), \\
& v_{4}(k)=\sum_{l=k-h_{m}}^{k-1} r^{l} x^{t}(l) r_{1} x(l)+\sum_{l=k-\tau_{m}}^{k-1} r^{l} y^{t}(l) r_{2} y(l), \\
& v_{5}(k)=r^{h_{m}} \sum_{\theta=-h_{m}}^{-1} \sum_{l=k+\theta}^{k-1} r^{l} \eta_{1}^{t}(l) z_{1} \eta_{1}(l)+r^{\tau_{m}} \sum_{\theta=-\tau_{m}}^{-1} \sum_{l=k+\theta}^{k-1} r^{l} \eta_{2}^{t}(l) z_{2} \eta_{2}(l)
\end{aligned}
$$

and $\eta_{1}(l)=x(l+1)-x(l), \eta_{2}(l)=y(l+1)-y(l)$. 
Calculating the difference of $V(k)$ along the trajectories of system (2.7), we can obtain

$$
\begin{aligned}
\delta v_{1}(k)= & r^{k+1}[a x(k)+w f(y(k-\tau(k)))]^{t} p_{1}[a x(k)+w f(y(k-\tau(k)))] \\
& +r^{k+1}[b y(k)+v g(x(k-h(k)))]^{t} p_{2}[b y(k)+v g(x(k-h(k)))] \\
& -r^{k} x^{t}(k) p_{1} x(k)-r^{k} y^{t}(k) p_{2} y(k) \\
= & r^{k}\left\{x^{t}(k)\left(r a^{t} p_{1} a-p_{1}\right) x(k)+2 r x^{t}(k) a^{t} p_{1} w f(y(k-\tau(k)))\right. \\
& \quad+r f^{t}(y(k-\tau(k))) w^{t} p_{1} w f(y(k-\tau(k)))+y^{t}(k)\left(r b^{t} p_{2} b-p_{2}\right) y(k) \\
& \left.\quad+2 r y^{t}(k) b^{t} p_{2} v g(x(k-h(k)))+r g^{t}(x(k-h(k))) v^{t} p_{2} v g(x(k-h(k)))\right\},
\end{aligned}
$$

$$
\begin{aligned}
\delta v_{3}(k)= & \left(h_{m}-h_{m}\right) r^{k} x^{t}(k) q_{1} x(k)+\left(\tau_{m}-\tau_{m}\right) r^{k} y^{t}(k) q_{2} y(k) \\
& -\sum_{l=k+1-h_{m}}^{k-h_{m}} r^{l} x^{t}(l) q_{1} x(l)-\sum_{l=k+1-\tau_{m}}^{k-\tau_{m}} r^{l} y^{t}(l) q_{2} y(l),
\end{aligned}
$$

$$
\begin{aligned}
\delta v_{4}(k)= & r^{k} x^{t}(k) r_{1} x(k)-r^{k-h_{m}} x^{t}\left(k-h_{m}\right) r_{1} x\left(k-h_{m}\right) \\
& +r^{k} y^{t}(k) r_{2} y(k)-r^{k-\tau_{m}} y^{t}\left(k-\tau_{m}\right) r_{2} y\left(k-\tau_{m}\right),
\end{aligned}
$$

$$
\begin{aligned}
\delta v_{5}(k)= & r^{k} r^{h_{m}} h_{m} \eta_{1}^{t}(k) z_{1} \eta_{1}(k)-r^{h_{m}} \sum_{l=k-h_{m}}^{k-1} r^{l} \eta_{1}^{t}(l) z_{1} \eta_{1}(l) \\
& +r^{k} r^{\tau_{m}} \tau_{m} \eta_{2}^{t}(k) z_{2} \eta_{2}(k)-r^{\tau_{m}} \sum_{l=k-\tau_{m}}^{k-1} r^{l} \eta_{2}^{t}(l) z_{2} \eta_{2}(l) \\
\leq & r^{k} r^{h_{m}} h_{m}[(a-i) x(k)+w f(y(k-\tau(k)))]^{t} z_{1}[(a-i) x(k)+w f(y(k-\tau(k)))] \\
& +r^{k} r^{\tau_{m}} \tau_{m}[(b-i) y(k)+v g(x(k-h(k)))]^{t} z_{2}[(b-i) y(k)+v g(x(k-h(k)))] \\
& -r^{k} \sum_{l=k-h(k)}^{k-1} \eta_{1}^{t}(l) z_{1} \eta_{1}(l)-r^{k} \sum_{l=k-h_{m}}^{k-h(k)} \eta_{1}^{t}(l) z_{1} \eta_{1}(l) \\
& -r^{k} \sum_{l=k-\tau(k)}^{k-1} \eta_{2}^{t}(l) z_{2} \eta_{2}(l)-r^{k} \sum_{l=k-\tau_{m}}^{k-\tau(k)} \eta_{2}^{t}(l) z_{2} \eta_{2}(l) .
\end{aligned}
$$


By Lemma 2.2, we have

$$
\begin{aligned}
-\sum_{l=k-h(k)}^{k-1} \eta_{1}^{t}(l) z_{1} \eta_{1}(l) & \leq 2 \sum_{l=k-h(k)}^{k-1} \eta_{1}^{t}(l) l^{t} \xi(k)+\sum_{l=k-h(k)}^{k-1} \xi^{t}(k) l z_{1}^{-1} l^{t} \xi(k) \\
& =2\left[x^{t}(k)-x^{t}(k-h(k))\right] l^{t} \xi(k)+h(k) \xi^{t}(k) l z_{1}^{-1} l^{t} \xi(k) \\
& \leq \xi^{t}(k)\left(\psi_{1} l^{t}+l \psi_{1}\right) \xi(k)+h_{m} \xi^{t}(k) l z_{1}^{-1} l^{t} \xi(k) \\
-\sum_{l=k-h_{m}}^{k-h(k)} \eta_{1}^{t}(l) z_{1} \eta_{1}(l) & \leq 2 \sum_{l=k-h_{m}}^{k-h(k)} \eta_{1}^{t}(l) m^{t} \xi(k)+\sum_{l=k-h_{m}}^{k-h(k)} \xi^{t}(k) m z_{1}^{-1} m^{t} \xi(k) \\
& =2\left[x^{t}(k-h(k))-x^{t}\left(k-h_{m}\right)\right] m^{t} \xi(k)+\left(h_{m}-h(k)\right) \xi^{t}(k) m z_{1}^{-1} m^{t} \xi(k) \\
& \leq \xi^{t}(k)\left(\psi_{2} m^{t}+m \psi_{2}\right) \xi(k)+\left(h_{m}-h_{m}\right) \xi^{t}(k) m z_{1}^{-1} m^{t} \xi(k) \\
-\sum_{l=k-\tau(k)}^{k-1} \eta_{2}^{t}(l) z_{2} \eta_{2}(l) & \leq 2 \sum_{l=k-\tau(k)}^{k-1} \eta_{2}^{t}(l) n^{t} \xi(k)+\sum_{l=k-\tau(k)}^{k-1} \xi^{t}(k) n z_{2}^{-1} n^{t} \xi(k) \\
& =2\left[y^{t}(k)-y^{t}(k-\tau(k))\right] n^{t} \xi(k)+\tau(k) \xi^{t}(k) n z_{2}^{-1} n^{t} \xi(k) \\
& \leq \xi^{t}(k)\left(\psi_{3} n^{t}+n \psi_{3}\right) \xi(k)+\tau_{m} \xi^{t}(k) n z_{2}^{-1} n^{t} \xi(k) \\
& \leq \xi^{t}(k)\left(\psi_{4} t^{t}+t \psi_{4}\right) \xi(k)+\left(\tau_{m}-\tau_{m}\right) \xi^{t}(k) t z_{2}^{-1} t^{t} \xi(k) \\
-\sum_{l=k-\tau_{m}}^{k} \eta_{2}^{t}(l) z_{2} \eta_{2}(l) & \leq 2 \sum_{l=k-\tau_{m}}^{k-\tau(k)} \eta_{2}^{t}(l) t^{t} \xi(k)+\sum_{l=k-\tau_{m}}^{k-\tau(k)} \xi^{t}(k) t z_{2}^{-1} t^{t} \xi(k) \\
& 2\left[y^{t}(k-\tau(k))-y^{t}\left(k-\tau_{m}\right)\right] t^{t} \xi(k)+\left(\tau_{m}-\tau(k)\right) \xi^{t}(k) t z_{2}^{-1} t^{t} \xi(k) \\
k-\tau(k) & \\
&
\end{aligned}
$$

where

$$
\begin{aligned}
& l=\left[\begin{array}{llllllll}
l_{1}^{t} & l_{2}^{t} & l_{3}^{t} & l_{4}^{t} & l_{5}^{t} & l_{6}^{t} & l_{7}^{t} & \left.l_{8}^{t}\right]^{t},
\end{array},\right. \\
& m=\left[\begin{array}{llllllll}
m_{1}^{t} & m_{2}^{t} & m_{3}^{t} & m_{4}^{t} & m_{5}^{t} & m_{6}^{t} & m_{7}^{t} & m_{8}^{t}
\end{array}\right]^{t} \text {, } \\
& n=\left[\begin{array}{llllllll}
n_{1}^{t} & n_{2}^{t} & n_{3}^{t} & n_{4}^{t} & n_{5}^{t} & n_{6}^{t} & n_{7}^{t} & n_{8}^{t}
\end{array}\right]^{t}, \\
& t=\left[\begin{array}{llllllll}
t_{1}^{t} & t_{2}^{t} & t_{3}^{t} & t_{4}^{t} & t_{5}^{t} & t_{6}^{t} & t_{7}^{t} & t_{8}^{t}
\end{array}\right]^{t}, \\
& \psi_{1}=\left[\begin{array}{llllllll}
i & 0 & 0 & 0 & 0 & -i & 0 & 0
\end{array}\right]^{t}, \\
& \psi_{2}=\left[\begin{array}{llllllll}
0 & 0 & 0 & 0 & 0 & i & -i & 0
\end{array}\right]^{t}, \\
& \psi_{3}=\left[\begin{array}{llllllll}
0 & -i & 0 & 0 & i & 0 & 0 & 0
\end{array}\right]^{t}, \\
& \psi_{4}=\left[\begin{array}{llllllll}
0 & i & -i & 0 & 0 & 0 & 0 & 0
\end{array}\right]^{t},
\end{aligned}
$$


and $\xi(k)=\left[x^{T}(k) y^{T}(k-\tau(k)) y^{T}\left(k-\tau_{M}\right) f^{T}(y(k-\tau(k))) y^{T}(k) x^{T}(k-h(k)) x^{T}\left(k-h_{M}\right) g^{T}(x(k-\right.$ $h(k)))]$. By inequalities (2.5), it is well known that there exist positive diagonally matrices $D_{i} \geq 0(i=1,2)$ such that the following inequalities hold:

$$
\begin{aligned}
& r^{k}\left[y^{t}(t-\tau(t)) d_{1} y(t-\tau(t))-f^{t}(y(t-\tau(t))) l_{1}^{-1} d_{1} l_{1}^{-1} f(y(t-\tau(t)))\right] \geq 0, \\
& r^{k}\left[x^{t}(t-h(t)) d_{2} x(t-h(t))-g^{t}(x(t-h(t))) l_{2}^{-1} d_{2} l_{2}^{-1} g(x(t-h(t)))\right] \geq 0,
\end{aligned}
$$

where $L_{1}=\operatorname{diag}\left\{l_{11}, l_{12}, \ldots, l_{1 m}\right\}, L_{2}=\operatorname{diag}\left\{l_{21}, l_{22}, \ldots, l_{2 n}\right\}$.

Substituting (3.10)-(3.13) into (3.9), we have

$$
\begin{aligned}
\delta v(k) \leq & \delta v_{1}(k)+\delta v_{2}(k)+\delta v_{3}(k)+\delta v_{4}(k)+\delta v_{5}(k) \\
& \left.+r^{k}\left[y^{t}(t-\tau(t)) d_{1} y(t-\tau(t))\right)-f^{t}(y(t-\tau(t))) l_{1}^{-1} d_{1} l_{1}^{-1} f(y(t-\tau(t)))\right] \\
& \left.+r^{k}\left[x^{t}(t-h(t)) d_{2} x(t-h(t))\right)-g^{t}(x(t-h(t))) l_{2}^{-1} d_{2} l_{2}^{-1} g(x(t-h(t)))\right] \\
\leq & r^{k} \xi^{t}(k)\left[\omega+h_{m} l z_{1}^{-1} l^{t}+\left(h_{m}-h_{m}\right) m z_{1}^{-1} m^{t}+\tau_{m} n z_{2}^{-1} n^{t}+\left(\tau_{m}-\tau_{m}\right) t z_{2}^{-1} t^{t}\right] \xi(k),
\end{aligned}
$$

where $\Omega, L, M, N$, and $T$ are defined in Theorem 3.1. Thus, if LMI (3.1) holds, it can be concluded that $\Delta V(k)<0$ according to Schur complement, which implies that $V(k)<V(0)$. Note that

$$
\begin{aligned}
v_{1}(0)= & x^{t}(0) p_{1} x(0)+y^{t}(0) p_{2} y(0) \\
\leq & \lambda_{m}\left(p_{1}\right) \sup _{s \in n\left[-h_{m}, 0\right]}\|\phi(s)\|^{2}+\lambda_{m}\left(p_{2}\right) \sup _{s \in n\left[-\tau_{m}, 0\right]}\|\psi(s)\|^{2}, \\
v_{2}(0)= & \sum_{l=-h(0)}^{-1} r^{l} x^{t}(l) q_{1} x(l)+\sum_{l=-\tau(0)}^{-1} r^{l} y^{t}(l) q_{2} y(l) \\
\leq & \lambda_{m}\left(q_{1}\right) \frac{1-r^{-h_{m}}}{r-1} \sup _{s \in n\left[-h_{m}, 0\right]}\|\phi(s)\|^{2}+\lambda_{m}\left(q_{2}\right) \frac{1-r^{-\tau_{m}}}{r-1} \sup _{s \in n\left[-\tau_{m}, 0\right]}\|\psi(s)\|^{2}, \\
v_{3}(0)= & \sum_{\theta=-h_{m}+1}^{-1} \sum_{l=\theta}^{-1} r^{l} x^{t}(l) q_{1} x(l)+\sum_{\theta=-\tau_{m}+1}^{-\tau_{m}} \sum_{l=\theta}^{-1} r^{l} y^{t}(l) q_{2} y(l) \\
\leq & \left(h_{m}-h_{m}\right) \sum_{l=h_{m}}^{-1} r^{l} x^{t}(l) q_{1} x(l)+\left(\tau_{m}-\tau_{m}\right) \sum_{l=\tau_{m}}^{-1} r^{l} y^{t}(l) q_{2} y(l) \\
\leq & \left(h_{m}-h_{m}\right) \lambda_{m}\left(q_{1}\right) \frac{1-r^{-h_{m}}}{r-1} \sup _{s \in n\left[-h_{m}, 0\right]}\|\psi(s)\|^{2} \\
& +\left(\tau_{m}-\tau_{m}\right) \lambda_{m}\left(q_{2}\right) \frac{1-r^{-\tau_{m}}}{r-1} \sup _{s \in n\left[-\tau_{m}, 0\right]}\|\psi(s)\|^{2},
\end{aligned}
$$




$$
\begin{aligned}
v_{4}(0)= & \sum_{l=-h_{m}}^{-1} r^{l} x^{t}(l) r_{1} x(l)+\sum_{l=-\tau_{m}}^{-1} r^{l} y^{t}(l) r_{2} y(l) \\
\leq & \lambda_{m}\left(r_{1}\right) \frac{1-r^{-h_{m}}}{r-1} \sup _{s \in n\left[-h_{m}, 0\right]}\|\phi(s)\|^{2}+\lambda_{m}\left(r_{2}\right) \frac{1-r^{-\tau_{m}}}{r-1} \sup _{s \in n\left[-\tau_{m}, 0\right]}\|\psi(s)\|^{2}, \\
v_{5}(0)= & r^{h_{m}} \sum_{\theta=-h_{m}}^{-1} \sum_{l=\theta}^{-1} r^{l} \eta_{1}^{t}(l) z_{1} \eta_{1}(l)+r^{\tau_{m}} \sum_{\theta=-\tau_{m}}^{-1} \sum_{l=\theta}^{1} r^{l} \eta_{2}^{t}(l) z_{2} \eta_{2}(l) \\
\leq & r^{h_{m}} h_{m} \lambda_{m}\left(z_{1}\right) \sum_{l=-h_{m}} r^{l} \eta_{1}^{t}(l) \eta_{1}(l)+r^{\tau_{m}} \tau_{m} \lambda_{m}\left(z_{2}\right) \sum_{l=-\tau_{m}}^{-1} r^{l} \eta_{2}^{t}(l) \eta_{2}(l) \\
\leq & r^{h_{m}} h_{m} \lambda_{m}\left(z_{1}\right) \frac{1-r^{-h_{m}}}{r-1} \sup _{s \in n\left[-h_{m},-1\right]}\left\|\eta_{1}(s)\right\|^{2} \\
& +r^{\tau_{m}} \tau_{m} \lambda_{m}\left(z_{2}\right) \frac{1-r^{-\tau_{m}}}{r-1} \sup _{s \in n\left[-\tau_{m},-1\right]}\left\|\eta_{2}(s)\right\|^{2}
\end{aligned}
$$

$$
\begin{aligned}
\sup _{s \in n\left[-h_{m},-1\right]}\left\|\eta_{1}(s)\right\|^{2} & =\sup _{s \in n\left[-h_{m},-1\right]}\|x(s+1)-x(s)\|^{2} \\
& \leq 2 \sup _{s \in n\left[-h_{m},-1\right]}\left(\|x(s+1)\|^{2}+\|x(s)\|^{2}\right) \\
& \leq 4 \sup _{s \in n\left[-h_{m}, 0\right]}\|\phi(s)\|^{2}, \\
\sup _{s \in n\left[-\tau_{m},-1\right]}\left\|\eta_{2}(s)\right\|^{2} & =\sup _{s \in n\left[-\tau_{m},-1\right]}\|y(s+1)-y(s)\|^{2} \\
& \leq 2 \sup _{s \in n\left[-\tau_{m},-1\right]}\left(\|y(s+1)\|^{2}+\|y(s)\|^{2}\right) \\
& \leq 4 \sup _{s \in n\left[-\tau_{m}, 0\right]}\|\psi(s)\|^{2} .
\end{aligned}
$$

Substituting (3.22), (3.23) into (3.21), and combining (3.17)-(3.21), then we have

$$
V(0)=\sum_{i=1}^{5} V_{i}(0) \leq \rho_{1} \sup _{s \in N\left[-h_{M}, 0\right]}\|\phi(s)\|^{2}+\rho_{2} \sup _{s \in N\left[-\tau_{M}, 0\right]}\|\psi(s)\|^{2},
$$

where

$$
\begin{aligned}
& \rho_{1}=\lambda_{m}\left(p_{1}\right)+\left[\left(h_{m}-h_{m}+1\right) \lambda_{m}\left(q_{1}\right)+\lambda_{m}\left(r_{1}\right)+4 r^{h_{m}} h_{m} \lambda_{m}\left(z_{1}\right)\right] \frac{1-r^{-h_{m}}}{r-1} \\
& \rho_{2}=\lambda_{m}\left(p_{2}\right)+\left[\left(\tau_{m}-\tau_{m}+1\right) \lambda_{m}\left(q_{2}\right)+\lambda_{m}\left(r_{2}\right)+4 r^{\tau_{m}} \tau_{m} \lambda_{m}\left(z_{2}\right)\right] \frac{1-r^{-\tau_{m}}}{r-1} .
\end{aligned}
$$


On the other hand,

$$
V(k) \geq r^{k} x^{T}(k) P_{1} x(k)+r^{k} y^{T}(k) P_{2} y(k) \geq r^{k}\left\{\lambda_{m}\left(P_{1}\right)\|x(k)\|^{2}+\lambda_{m}\left(P_{2}\right)\|y(k)\|^{2}\right\} .
$$

Therefore, we can obtain

$$
\|x(k)\|^{2}+\|y(k)\|^{2} \leq \frac{\alpha}{\beta}\left(\sup _{s \in N\left[-h_{M}, 0\right]}\|\phi(s)\|^{2}+\sup _{s \in N\left[-\tau_{M}, 0\right]}\|\psi(s)\|^{2}\right) r^{-k}
$$

where $\alpha=\max \left\{\rho_{1}, \rho_{2}\right\}, \beta=\min \left\{\lambda_{m}\left(P_{1}\right), \lambda_{m}\left(P_{2}\right)\right\}$. Obviously, $\alpha / \beta>1$, by Definition 2.1 , the system (2.7) or (2.1) is globally exponentially stable.

Remark 3.2. Based on the linear matrix inequality (LMI) technique and Lyapunov stability theory, the exponential stability for discrete-time delayed BAM neural network (2.1) was investigated in [26-28]. However, it should be noted that the results in [26] are based on the unreasonably severe constraints on the delay functions $1<\tau(k+1)<1+\tau(k), 1<h(k+1)<1+$ $h(k)$, and the results in $[27,28]$ are based on the simple Lyapunov functionals. In this paper, in order to obtain the less conservative stability criterion, the novel Lyapunov functional $V(k)$ is employed, which contains $V_{4}(k), V_{5}(k)$, and more general than the traditional ones [26-28]. Moreover, some slack matrices, which bring much flexibility in solving LMI, are introduced in this paper.

Remark 3.3. By setting $r=1$ in Theorem 3.1, we can obtain the global asymptotic stability criterion of discrete-time BAM neural network (2.7).

Remark 3.4. Theorem 3.1 in this paper depends on both the delay upper bounds $\tau_{M}, h_{M}$ and the delay intervals $\tau_{M}-\tau_{m}$ and $h_{M}-h_{m}$.

Remark 3.5. The proposed method in this paper can be generalized to more complex neural networks, such as delayed discrete-time BAM neural networks with parameter uncertainties $[30,31]$ and stochastic perturbations [30-32], delayed interval discrete-time BAM neural networks [28], and discrete-time analogues of BAM neural networks with mixed delays $[32,33]$.

\section{Numerical examples}

Example 4.1. Consider the delayed discrete-time BAM neural network (2.7) with the following parameters:

$$
A=\left[\begin{array}{cc}
0.8 & 0 \\
0 & 0.9
\end{array}\right], \quad B=\left[\begin{array}{cc}
0.5 & 0 \\
0 & 0.4
\end{array}\right], \quad W=\left[\begin{array}{cc}
0.1 & -0.01 \\
-0.2 & -0.1
\end{array}\right], \quad V=\left[\begin{array}{cc}
0.15 & 0 \\
-0.2 & 0.1
\end{array}\right]
$$

The activation functions satisfy Assumptions (A1) and (A2) with

$$
L_{1}=\left[\begin{array}{ll}
1 & 0 \\
0 & 1
\end{array}\right], \quad L_{2}=\left[\begin{array}{ll}
1 & 0 \\
0 & 1
\end{array}\right]
$$


Table 1: Maximum allowable delay bounds for Example 4.1.

\begin{tabular}{lcccccccc}
\hline$\tau_{m}=h_{m}$ & 2 & 4 & 6 & 8 & 10 & 15 & 20 & 25 \\
$\tau_{M}=h_{M}[27,28]$ & 6 & 8 & 10 & 12 & 14 & 19 & 24 & 29 \\
$\tau_{M}=h_{M}$ Theorem 3.1 & 11 & 12 & 13 & 15 & 16 & 21 & 25 & 30 \\
\hline
\end{tabular}

For this example, by Theorem 3.1 in this paper with $r=1$, we can obtain some maximum allowable delay bounds for guaranteeing the asymptotic stability of this system. For a comparison with [27, Theorem 1] and in [28, Corollary 1], we made Table 1. For this example, it is obvious that our result is less conservative than those in $[27,28]$.

Example 4.2 (see [27]). Consider the delayed discrete-time BAM NN (2.7) with the following parameters:

$$
A=\left[\begin{array}{cc}
\frac{1}{5} & 0 \\
0 & \frac{1}{5}
\end{array}\right], \quad B=\left[\begin{array}{cc}
\frac{1}{10} & 0 \\
0 & \frac{1}{10}
\end{array}\right], \quad W=\left[\begin{array}{cc}
0 & \frac{1}{8} \\
\frac{1}{8} & 0
\end{array}\right], \quad V=\left[\begin{array}{cc}
-\frac{1}{20} & 0 \\
0 & -\frac{1}{20}
\end{array}\right]
$$

The activation functions satisfy Assumptions (A1) and (A2) with

$$
L_{1}=\left[\begin{array}{ll}
1 & 0 \\
0 & 1
\end{array}\right], \quad L_{2}=\left[\begin{array}{ll}
1 & 0 \\
0 & 1
\end{array}\right]
$$

Now, we assume $\tau(k)=h(k)=4-2 \sin ((\pi / 2) k), r=1.55$. It is obvious that $2 \leq \tau(k)=h(k) \leq 6$, that is, $h_{m}=\tau_{m}=2, h_{M}=\tau_{m}=6$. In this case, it is found that the exponential conditions proposed in $[27,28]$ is not satisfied. However, it can be concluded that this system is globally exponentially stable by using Theorem 3.1 in this paper. If we assume $h_{m}=\tau_{m}=\tau_{M}=2, r=2.7$. Using [27, Theorem 1] and [28, Corollary 1], the achieved maximum allowable delays for guaranteeing the globally exponentially stable of this system are $h_{M}=3, h_{M}=3$, respectively. However, we can obtain the delay bound $h_{M}=4$ by using Theorem 3.1 in this paper. For this example, it is seen that our result improve some existing results $[27,28]$.

\section{Conclusion}

In this paper, we consider the delay-dependent exponential stability for discrete-time BAM neural networks with time-varying delays. Based on the Lyapunov stability theory and linear matrix inequality technique, the novel delay-dependent stability criterion is obtained. Two numerical examples show that the proposed stability condition in this paper is less conservative than previously established ones.

\section{Acknowledgments}

The authors would like to thank the Associate Editor and the anonymous reviewers for their constructive comments and suggestions to improve the quality of the paper. This work was supported by the National Natural Science Foundation of China (no. 60643003) and the Natural Science Foundation of Henan Education Department (no. 2007120005). 


\section{References}

[1] B. Kosko, "Adaptive bidirectional associative memories," Applied Optics, vol. 26, no. 23, pp. 49474960, 1987.

[2] B. Kosko, "Bidirectional associative memories," IEEE Transactions on Systems, Man and Cybernetics, vol. 18, no. 1, pp. 49-60, 1988.

[3] J. Cao and L. Wang, "Exponential stability and periodic oscillatory solution in BAM networks with delays," IEEE Transactions on Neural Networks, vol. 13, no. 2, pp. 457-463, 2002.

[4] J. Cao and M. Dong, "Exponential stability of delayed bi-directional associative memory networks," Applied Mathematics and Computation, vol. 135, no. 1, pp. 105-112, 2003.

[5] Y. Li, "Existence and stability of periodic solution for BAM neural networks with distributed delays," Applied Mathematics and Computation, vol. 159, no. 3, pp. 847-862, 2004.

[6] X. Huang, J. Cao, and D.-S. Huang, "LMI-based approach for delay-dependent exponential stability analysis of BAM neural networks," Chaos, Solitons Fractals, vol. 24, no. 3, pp. 885-898, 2005.

[7] S. Arik, "Global asymptotic stability analysis of bidirectional associative memory neural networks with time delays," IEEE Transactions on Neural Networks, vol. 16, no. 3, pp. 580-586, 2005.

[8] M. Jiang, Y. Shen, and X. Liao, "Global stability of periodic solution for bidirectional associative memory neural networks with varying-time delay," Applied Mathematics and Computation, vol. 182, no. 1, pp. 509-520, 2006.

[9] J. H. Park, "A novel criterion for global asymptotic stability of BAM neural networks with time delays," Chaos, Solitons Fractals, vol. 29, no. 2, pp. 446-453, 2006.

[10] X. Lou and B. Cui, "On the global robust asymptotic stability of BAM neural networks with timevarying delays," Neurocomputing, vol. 70, no. 1-3, pp. 273-279, 2006.

[11] J. H. Park, "Robust stability of bidirectional associative memory neural networks with time delays," Physics Letters A, vol. 349, no. 6, pp. 494-499, 2006.

[12] J. Cao, D. W. C. Ho, and X. Huang, "LMI-based criteria for global robust stability of bidirectional associative memory networks with time delay," Nonlinear Analysis: Theory, Methods Applications, vol. 66, no. 7, pp. 1558-1572, 2007.

[13] D. W. C. Ho, J. Liang, and J. Lam, “Global exponential stability of impulsive high-order BAM neural networks with time-varying delays," Neural Networks, vol. 19, no. 10, pp. 1581-1590, 2006.

[14] L. Sheng and H. Yang, "Novel global robust exponential stability criterion for uncertain BAM neural networks with time-varying delays," Chaos, Solitons Fractals. In press.

[15] J. H. Park, S. M. Lee, and O. M. Kwon, “On exponential stability of bidirectional associative memory neural networks with time-varying delays," Chaos, Solitons Fractals. In press.

[16] Y. Wang, "Global exponential stability analysis of bidirectional associative memory neural networks with time-varying delays," Nonlinear Analysis: Real World Applications. In press.

[17] Y. Liu, Z. Wang, A. Serrano, and X. Liu, "Discrete-time recurrent neural networks with time-varying delays: exponential stability analysis," Physics Letters A, vol. 362, no. 5-6, pp. 480-488, 2007.

[18] S. Hu and J. Wang, "Global stability of a class of discrete-time recurrent neural networks," IEEE Transactions on Circuits and Systems I, vol. 49, no. 8, pp. 1104-1117, 2002.

[19] S. Mohamad and K. Gopalsamy, "Exponential stability of continuous-time and discrete-time cellular neural networks with delays," Applied Mathematics and Computation, vol. 135, no. 1, pp. 17-38, 2003.

[20] W. Xiong and J. Cao, "Global exponential stability of discrete-time Cohen-Grossberg neural networks," Neurocomputing, vol. 64, pp. 433-446, 2005.

[21] L. Wang and Z. Xu, "Sufficient and necessary conditions for global exponential stability of discretetime recurrent neural networks," IEEE Transactions on Circuits and Systems I, vol. 53, no. 6, pp. 13731380, 2006.

[22] W.-H. Chen, X. Lu, and D.-Y. Liang, "Global exponential stability for discrete-time neural networks with variable delays," Physics Letters A, vol. 358, no. 3, pp. 186-198, 2006.

[23] Q. Song and Z. Wang, "A delay-dependent LMI approach to dynamics analysis of discrete-time recurrent neural networks with time-varying delays," Physics Letters A, vol. 368, no. 1-2, pp. 134-145, 2007.

[24] B. Zhang, S. Xu, and Y. Zou, "Improved delay-dependent exponential stability criteria for discretetime recurrent neural networks with time-varying delays," Neurocomputing. In press. 
[25] J. Liang and J. Cao, "Exponential stability of continuous-time and discrete-time bidirectional associative memory networks with delays," Chaos, Solitons

Fractals, vol. 22, no. 4, pp. 773-785, 2004.

[26] J. Liang, J. Cao, and D. W. C. Ho, “Discrete-time bidirectional associative memory neural networks with variable delays," Physics Letters A, vol. 335, no. 2-3, pp. 226-234, 2005.

[27] X.-G. Liu, M.-L. Tang, R. Martin, and X.-B. Liu, "Discrete-time BAM neural networks with variable delays," Physics Letters A, vol. 367, no. 4-5, pp. 322-330, 2007.

[28] M. Gao and B. Cui, "Global robust exponential stability of discrete-time interval BAM neural networks with time-varying delays," Applied Mathematical Modelling. In press.

[29] M. S. Mahmoud, Resilient Control of Uncertain Dynamical Systems, vol. 303 of Lecture Notes in Control and Information Sciences, Springer, Berlin, Germany, 2004.

[30] Z. Wang, S. Lauria, J. Fang, and X. Liu, "Exponential stability of uncertain stochastic neural networks with mixed time-delays," Chaos, Solitons Fractals, vol. 32, no. 1, pp. 62-72, 2007.

[31] Z. Wang, H. Shu, J. Fang, and X. Liu, "Robust stability for stochastic Hopfield neural networks with time delays," Nonlinear Analysis: Real World Applications, vol. 7, no. 5, pp. 1119-1128, 2006.

[32] Z. Wang, Y. Liu, M. Li, and X. Liu, "Stability analysis for stochastic Cohen-Grossberg neural networks with mixed time delays," IEEE Transactions on Neural Networks, vol. 17, no. 3, pp. 814-820, 2006.

[33] Z. Wang, Y. Liu, and X. Liu, "On global asymptotic stability of neural networks with discrete and distributed delays," Physics Letters A, vol. 345, no. 4-6, pp. 299-308, 2005. 


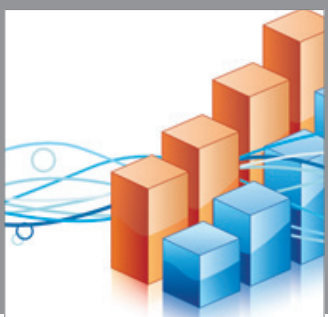

Advances in

Operations Research

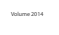

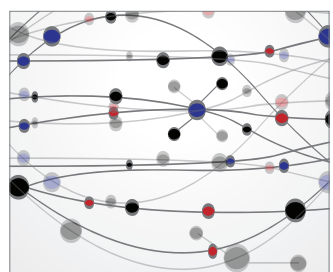

\section{The Scientific} World Journal
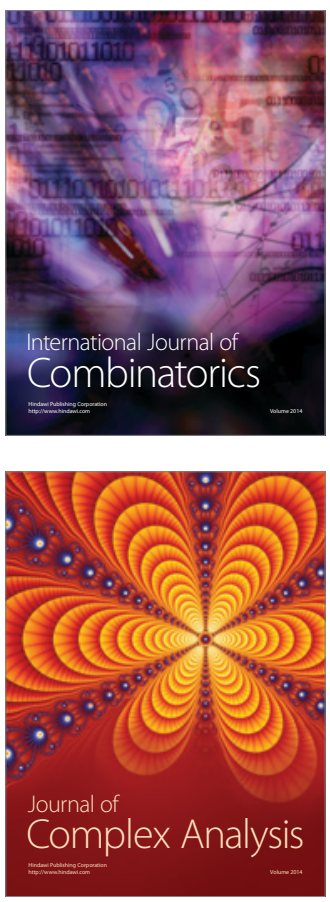

International Journal of

Mathematics and

Mathematical

Sciences
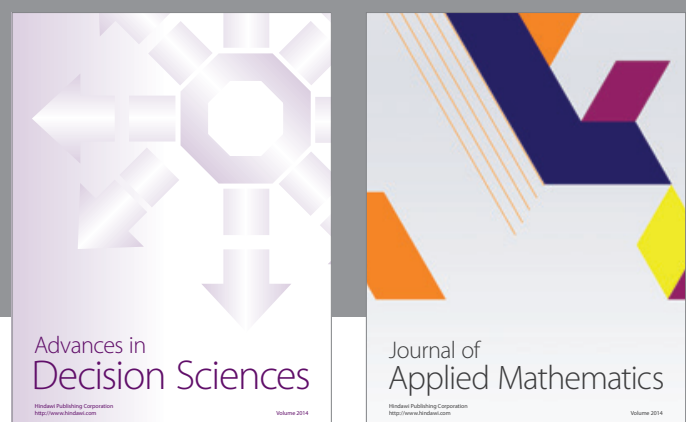

Journal of

Applied Mathematics
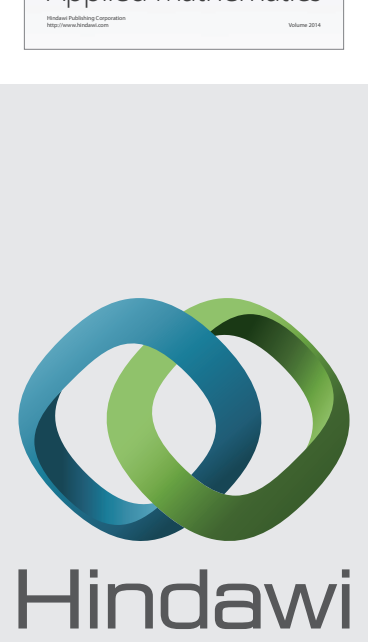

Submit your manuscripts at http://www.hindawi.com
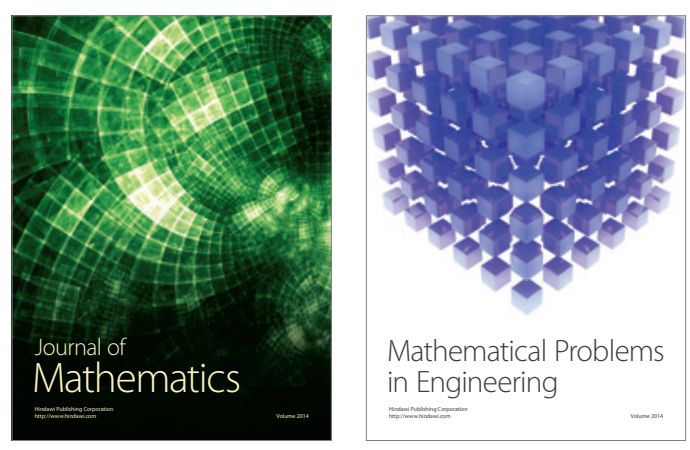

Mathematical Problems in Engineering
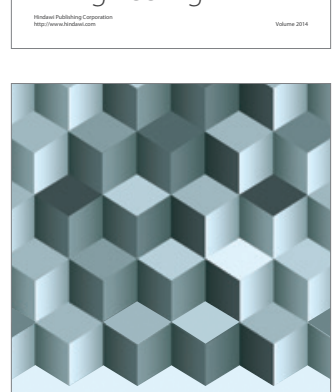

Journal of

Function Spaces
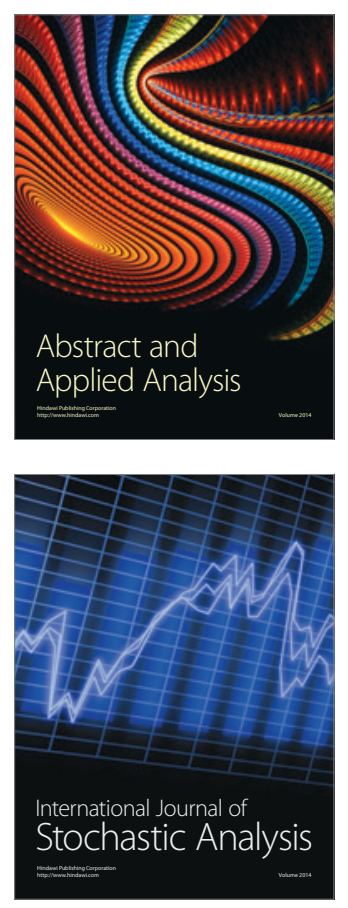

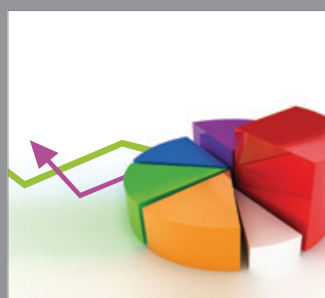

ournal of

Probability and Statistics

Promensencen
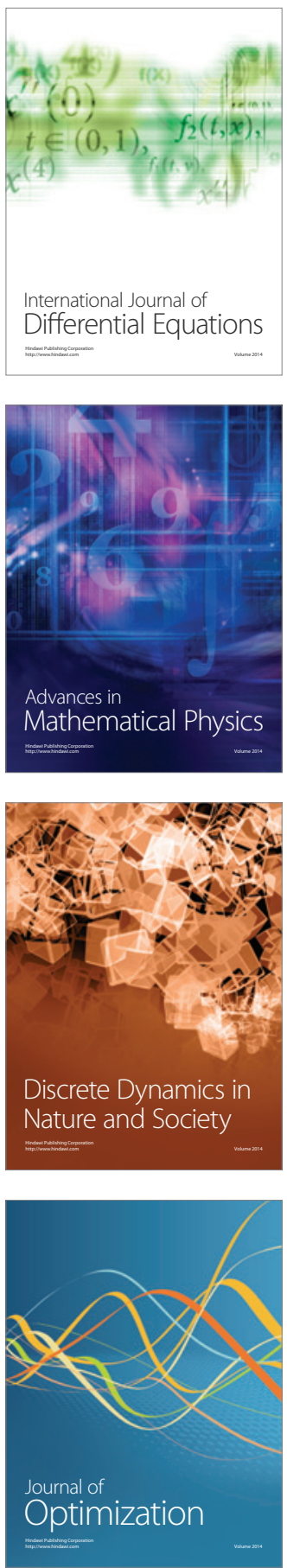